\title{
A Family of High Continuity Subdivision Schemes Based on Probability Distribution
}

\author{
MUHAMMAD ASGHAR*, MUHAMMAD JAVED IQBAL**, AND GHULAM MUSTAFA* \\ RECEIVED ON 01.11.2017 ACCEPTED ON 25.05.2018
}

\begin{abstract}
Subdivision schemes are famous for the generation of smooth curves and surfaces in CAGD (Computer Aided Geometric Design). The continuity is an important property of subdivision schemes. Subdivision schemes having high continuity are always required for geometric modeling. Probability distribution is the branch of statistics which is used to find the probability of an event. We use probability distribution in the field of subdivision schemes. In this paper, a simplest way is introduced to increase the continuity of subdivision schemes. A family of binary approximating subdivision schemes with probability parameter p is constructed by using binomial probability generating function. We have derived some family members and analyzed the important properties such as continuity, Holder regularity, degree of generation, degree of reproduction and limit stencils. It is observed that, when the probability parameter $p=1 / 2$, the family of subdivision schemes have maximum continuity, generation degree and Holder regularity. Comparison shows that our proposed family has high continuity as compare to the existing subdivision schemes. The proposed family also preserves the shape preserving property such as convexity preservation. Subdivision schemes give negatively skewed, normal and positively skewed behavior on convex data due to the probability parameter. Visual performances of the family are also presented.
\end{abstract}

Key Words: Binary, Approximating Subdivision Schemes, Binomial Distribution, Continuity Analysis, Convexity Preservation.

\section{INTRODUCTION}

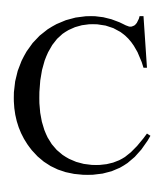

AGD concerns with the mathematical description of shape for use in computer graphics. Subdivision schemes are iterative

formulas for generation of smooth curves and surfaces. They give effective approaches to generate smooth curves and surfaces from discrete arrangement of control points.
Initially, De Rham [1] gave the idea of CAGD with geometry. After that Chaikin [2] introduced a procedure to generate curves from limited number of points. This algorithm was one of the first refinement algorithms to generate curves. Dyn et. al. [3] presented a subdivision scheme with tension parameter which is $\mathrm{C}^{1}$ continuous for a certain range of parameter. Mustafa and Liu [4] presented a new solid parametric subdivision scheme.

Authors E-Mail: (m.asgharmaths@gmail.com, javediqbalmp5@yahoo.com, ghulam.mustafa@iub.edu.pk) * Department of Mathematics, The Islamia University of Bahawalpur, Bahawalpur, Pakistan.

** Department of Mathematics, National College of Business Administration \& Economics, Sub-Campus Bahawalpur, Pakistan.

This is an open access article published by Mehran University Research Journal of Engineering and Technology, Jamshoro under the CC by 4.0 International License. 
Mustafa et. al. [5] proposed and analyzed the $m$-point binary approximating parametric subdivision scheme and compared the proposed scheme with existing schemes. Ghaffar and Mustafa [6] introduced new families of subdivision schemes for curve design. Mustafa et. al. [7] presented a family of (2n-1)-point binary approximating subdivision schemes with free parameters for describing curves. Ashraf et. al. [8] presented a new family of subdivision schemes by using a variant of LaneRiesenfeld algorithm.

Cao and Tan [9] presented and analyzed the important properties of new 5-point relaxed subdivision scheme. Their scheme produce $\mathrm{C}^{2}$ limit curve when $w \in\left(\frac{-3}{64}, \frac{1}{162}\right)$.

Mustafa et. al. [10] presented a proof of 6-point scheme. They said that the scheme produce $C^{2}$ and $C^{3}$ limit curve when $w \in[0,0425]$, and $w \in[0.0139,0.0143]$, respectively.

Bari et. al. [11] presented shape preserving subdivision schemes. Siddiqi and Noreen [12] presented the convexity preserving property of 6-point ternary interpolating subdivision scheme with the tension parameter. Tan et. al. [13] also discussed the convexity preserving property of 5-point binary scheme.

Zheng et. al. [14] presented a technique to increase the continuity of any subdivision scheme. They multiply the symbol of the scheme with $(1+z / 2)^{k}$ factor to get $C^{n+k}$ continuous subdivision schemes. But the technique used by them increase the complexity of the scheme. In this paper, we have presented a way to introduce a new family of subdivision schemes. Our technique is based on probability generating function of Binomial probability distribution. By using this technique one can able to increase the continuity, Holder regularity, degree of generation, degree of reproduction of any subdivision scheme without increasing the complexity of the scheme.

Probability is the chance of occurrence. A variable that shows the probabilities as the outcome of an experiment is called random variable. A list of probabilities associated to each value of random variable is called probability distribution and a function that is used for this purpose is called probability generating function. Binomial probability distribution was introduced by Bernoulli [15].

The paper is organized as follows. In Section 2, we construct a general symbol of family of binary approximating subdivision scheme. Complete analysis of some family members of proposed family is presented in Section 3. Convexity preservation is discussed in Section 4. Applications and comparison are presented in Section 5. Conclusions are drawn in Sections 6.

\section{CONSTRUCTION OFA FAMILY OF SCHEMES}

In this section, we will present a generalized symbol for the construction of a family of binary approximating subdivision schemes. For this, we consider a well-known 4-point scheme [16] given by:

$$
\begin{aligned}
& f_{2 i}^{k+1}=\frac{1}{384}\left(27 f_{i-1}^{k}+235 f_{i}^{k}+121 f_{i+1}^{k}+f_{i+2}^{k}\right) \\
& f_{2 i+1}^{k+1}=\frac{1}{384}\left(f_{i-1}^{k}+121 f_{i}^{k}+235 f_{i+1}^{k}+27 f_{i+2}^{k}\right)
\end{aligned}
$$

The Laurent polynomial of the scheme Equation (1) is:

$$
B(z)=\frac{1}{384}\left(1+27 z+121 z^{2}+235 z^{3}+235 z^{4}+121 z^{5}+27 z^{6}+z^{7}\right)
$$

Binomial probability generating function [17] is given as: 
$H_{n}(z)=\sum_{i=0}^{n}\left(\begin{array}{l}n \\ i\end{array}\right) p^{i}(1-p)^{n-i} z^{i}$

where $i=0,1,2, \ldots, n$.

Here $n$ is the number of trails, $p$ is the probability of success, $q=1-p$ is the probability of failure and also $p+$ $q=1$. For the construction of a family of binary approximating subdivision schemes, we multiply $B(z)$ and $H_{n}(\mathrm{z})$

$$
A_{n}(z)=\frac{1}{384}(1+z)^{5}\left(1+22 z+z^{2}\right) \sum_{i=0}^{n}\left(\begin{array}{l}
n \\
i
\end{array}\right) p^{i}(1-p)^{n-i} z^{i}
$$

We can easily derive symbol of the schemes by substituting $=1,2, \ldots$, in Equation (4) with $p \in(0,1)$. Before going onward to elaborate behavior and considerable properties of the proposed family of schemes $A_{n}=\left\{A_{n}: n\right.$ $=1,2, \ldots\}$, let us have a smart look at some members of this family.

\subsection{DERIVATION OF FAMILY MEMBERS}

Relaxed 4-Point Scheme (i.e. A) ): After substituting $n=1$ in Equation (4), we get the following symbol of relaxed 4point binary approximating subdivision scheme

$$
\begin{gathered}
A_{1}(z)=\frac{1}{384}\left[(1+p)+(27-26 p) z+(121-94 p) z^{2}+(235-114 p) z^{3}+235 z^{4}\right. \\
\left.+(121+114 p) z^{5}+(27+94 p) z^{6}+(1+26 p) z^{7}+p z^{8}\right]
\end{gathered}
$$

The scheme corresponding to Equation (5) is:

$$
\begin{aligned}
& f_{2 i}^{k+1}=\frac{1}{384}\left[(27-26 p) f_{i-2}^{k}+(235-114 p) f_{i-1}^{k}+(121+114 p) f_{i}^{k}+(1-26 p) f_{i+1}^{k}\right] \\
& f_{2 i+1}^{k+1}=\frac{1}{384}\left[(1-p) f_{i-2}^{k}+(121-94 p) f_{i-1}^{k}+235 f_{i}^{k}+(27+94 p) f_{i+1}^{k}+p f_{i+1}^{k}\right]
\end{aligned}
$$

5-Point Scheme (i.e. $\mathbf{A}_{2}$ ): By putting $n=2$ in Equation (4), we obtain the symbol of 5-point binary approximating subdivision scheme

$$
A_{2}(z)=\frac{1}{384}(1+z)^{5}\left(1+22 z+z^{2}\right)\left(p^{2}+2 p\right)(1-p) z+(1-p)^{2} z^{2}
$$

The scheme corresponding to Equation (7) is:

$$
\begin{gathered}
f_{2 i}^{k+1}=\frac{1}{384}\left[\left(25 p^{2}+2 p\right) f_{i-2}^{k}+\left(20 p^{2}+188 p+27\right) f_{i-1}^{k}+\left(-114 p^{2}+235\right)\right. \\
\left.f_{i}^{k}+\left(68 p^{2}-188 p+121\right) f_{i+1}^{k}+\left(p^{2}-2 p+1\right) f_{i+2}^{k}\right] \\
f_{2 i+1}^{k+1}=\frac{1}{384}\left[p^{2} f_{i-2}^{k}+\left(68 p^{2}+52 p+1\right) f_{i-1}^{k}+\left(-114 p^{2}+228 p+121\right)\right. \\
\left.f_{i}^{k}+\left(20 p^{2}-228 p+235\right) f_{i+1}^{k}+\left(25 p^{2}-52 p+27\right) f_{i+2}^{k}\right]
\end{gathered}
$$

Similarly by substituting $n=3,4,5, \ldots$, we obtain 6 -point relaxed, 6-point, 7-point relaxed subdivision schemes respectively.

\section{ANALYSIS OFTHE SCHEMES}

Aim of this section is to present the analysis of proposed family of binary approximating subdivision schemes. Here we only present the analysis of one family member $A_{2}$ of binary subdivision schemes. The analysis of rest of the schemes are similar.

We use Laurent polynomial (symbol) method [18] to calculate integer class continuity, degree of generation and degree of reproduction of the $A_{n}$ schemes. Moreover, Holder regularity analysis is done by using Riouls [19] method. Using [18], the subdivision scheme with symbol $A_{n}(z)$ reproduces polynomials of degree $d$ with respect to the parameterizations $\tau=A_{n}(1) / 2$ if and only if

$A_{n}^{k}(-1)=0$, and $A_{n}^{k}(1)=2 \prod_{j=0}^{k-1}(\tau-j), \mathrm{k}=0,1,2, \ldots, \mathrm{d}$

Polynomial reproduction of degree $d$ requires polynomial generation of degree $d$. 
Theorem-1: The 5-point binary subdivision scheme $A_{2}$ is $C^{4}$-continuous.

Proof: We re-write Equation (7) as:

$$
A_{2}(z)=\left(\frac{1+z}{2}\right)^{4} b(z)
$$

where

$b(z)=\frac{1}{24 z^{4}}\left[p^{2}+\left(21 p^{2}+2 p\right) z+\left(-22 p^{2}+44 p+1\right) z^{2}+\left(-22 p^{2}+23 p\right) z^{3}+\left(21 p^{2}-44 p+23\right) z^{4}+\left(p^{2}-2 p+1\right) z^{5}\right]$

Consider a difference scheme $S_{b}$ corresponding to $b(\mathrm{z})$ and $S_{c}$ corresponding to $c(z)$ obtained from $b(\mathrm{z})$, such that $b(\mathrm{z})=(1+\mathrm{z}) c(\mathrm{z})$ where

$c(z)=\frac{1}{24 z^{4}}\left[p^{2}+\left(20 p^{2}+2 p\right) z+\left(-42 p^{2}+42 p+1\right) z^{2}+\left(20 p^{2}-42 p+22\right) z^{2}+\left(p^{2}-2 p+1\right) z^{3}\right]$

To prove $\mathrm{C}^{4}$-continuity of the scheme $\mathrm{A}_{2}$, we need to show that $\mathrm{S}_{\mathrm{c}}$ is contractive. For contractiveness of $\mathrm{S}_{\mathrm{c}}$

$$
A_{n}^{k}(-1)=0, \text { and } A_{n}^{k}(1)=2 \prod_{j=0}^{k-1}(\tau-j), \quad k=0,1,2, \ldots, d
$$

The condition for $\left\|S_{c}\right\|_{\infty}<1$ is $\mathrm{p} \in(0,1)$. Therefore by [20], if $\left\|S_{c}\right\|_{\infty}<1$, then $\mathrm{c}(\mathrm{z})$ is contractive and $\mathrm{b}(\mathrm{z})$ is convergent. If $b(z)$ is convergent then the scheme Equation (8) is $\mathrm{C}^{4}$-continuous. Which completes the proof.

Remark-1: The 5-point binary approximating subdivision scheme Equation $(8)$ is $C^{6}$-continuous atp $=1 / 2$.

Theorem-2: The lower and upper bounds on the Hlder regularity of the scheme Equation (8) is 6.1256 at $p=1 / 2$.

Proof: From Equation (7), we have $b_{0}=1 / 12, b_{1}=22 / 12, b_{2}$ $=1 / 12, \mathrm{k}=7, \mathrm{~m}=2$ thus $1=0,1$ and $\mathrm{B}_{0}$ and $\mathrm{B}_{1}$ are the matrices with elements
$\left(\mathrm{B}_{0}\right)_{\mathrm{ij}}=\mathrm{b}_{2+\mathrm{i}-2 \mathrm{j}}$ and $\left(\mathrm{B}_{1}\right)_{\mathrm{ij}}=\mathrm{b}_{3+\mathrm{i}-2 \mathrm{j}}$

where $\mathrm{i}, \mathrm{j}=1$ and 2 . Thus $\mathrm{B}_{0}$ and $\mathrm{B}_{1}$ are given by

The eigenvalues of $\mathrm{B}_{0}$ and $\mathrm{B}_{1}$ are 1.8333 and 0.08333 . The norm infinity of $\mathrm{B}_{0}$ and $\mathrm{B}_{1}$ are 1.8333 and 1.8333 respectively. For bounds on Holder regularity, we calculate, where $\mu$ is joint spectral radius of the matrices of $\beta_{0}$ and $\beta_{1}$, that is, $\mu=\rho\left(B_{0}, B_{1}\right)$. Then by [19], lower bound and upper bound on Holder regularity are defined as:

So, $\max (1.8333,0.08333) \leq \mu \leq \max (1.8331,1.8333)$. So the lower and upper bounds on the Holder regularity is 6.1256. This completes the proof.

Theorem-3: The degree of the polynomial generation of 5 -point binary approximating subdivision scheme Equation (8) is 4.

Proof: By Equation (7), we can re-write the Laurent polynomial as:

$\left.\mathrm{A}_{2}(\mathrm{z})=1+\mathrm{z}\right)^{4+1} \mathrm{~b}_{1}(\mathrm{z})$

where

Hence by [18], generation degree is 4 .

Remark-2: At $p=1 / 2$, the degree of generation of the scheme Equation (8) is 6.

Theorem-4: The 5-point binary approximating subdivision scheme Equation (8) has linear reproduction with parametrization depends on the value of $p$.

Proof: By taking the first derivative of Equation (7) and put $\mathrm{z}=1$, we get $\mathrm{A}_{2}^{\prime}(1)=-2 \mathrm{p}^{2}-10 \mathrm{p}+9$. This implies that $\tau=1 / 2\left(-2 p^{2}-10 p+9\right)$, for different values of $p$, the scheme corresponding to thesymbol $\mathrm{A}_{2}$ has dual as well as primal parametrization. We can easily verify that first 
and second derivative of $\mathrm{A}_{2}$ at $\mathrm{z}=-1$ are equal to 0 . Further we can also verify Equation (9) for $\mathrm{k}=0$ and 1. This completes the proof.

In Table 1, we present the complete analysis of some family members of the proposed family of binary approximating subdivision schemes. Here we see that at $p=1 / 2$ the order of continuity and degree of generation have been increased. Moreover, we also present the Holder regularity analysis.

Table 1 shows the analysis of proposed family of schemes, here $\mathrm{n}, \mathrm{A}_{\mathrm{n}}, \mathrm{GD}, \mathrm{GD}_{1 / 2}, \mathrm{RD}, \mathrm{OC}, \mathrm{OC}_{1 / 2}$ and $\mathrm{HR}_{1 / 2}$, represent trial number, proposed schemes, degree of generation, degree of generation at $p=1 / 2$, degree of reproduction, order of continuity, order of continuity at $p=1 / 2$ and Holder regularity of proposed schemes at $p=1 / 2$ respectively.

\subsection{Limit Stencil}

The limit stencil is a way to obtain a point on the limit curve by using initial control points. The procedure for calculating the limit stencils is presented in [18]. In Table 2, we present the limit stencil of some family members of binary approximating subdivision schemes at $p=1 / 2$.

Table 2 shows the limit stencils of some family members of family of binary approximating subdivision schemes at $p=1 / 2$.

\section{CONVEXITY PRESERVATION}

In this section, we show the convexity preservation of the scheme $A_{2}$. It is clear from Fig. 1(a-c) that if initial control points are strictly convex. Then the limit curves generated by the scheme corresponding to $A_{2}$ show positively, normal and negatively skewed behavior on convex data for $p<1 / 2, p=1 / 2$ and $p>1 / 2$ respectively.

Theorem-5: Suppose that initial control points $\left\{p_{i}^{0}\right\}_{i \in Z}$ are strictly convex, i.e. $p_{i}^{0}, \forall i \in Z$. Let $d_{i}^{k}=2^{2 k+1}\left(f_{i-1}^{k}-2 f_{i}^{k}+f_{i+1}^{k}\right)$ be defined as second order differences, $r_{i}^{k}=\frac{d_{i+1}^{k}}{d_{i}^{k}}, R^{k}=\max \left\{r_{i}^{k}, \frac{1}{r_{i}^{k}}\right\}, \forall k \geq 0, k \in Z, i \in Z, \quad$ the parameter $p$ satisfies $0<\mathrm{p}<1$ and $\lambda \in Z$,

where

$\beta_{1}<\lambda<\beta_{2}$ and $\frac{1}{\lambda} \leq R^{0} \leq \lambda$

With

$\beta_{1}=\frac{1}{21 p^{2}-90 p+70}\left(22 p^{2}-44 p-13+\sqrt{p^{4}+92 p^{2}-66 p^{2}+1004 p+169}\right)$

TABLE 2. LIMIT STENCILS ANALYSIS OF FAMILY OF SUBDIVISION SCHEMES

\begin{tabular}{|c|c|}
\hline An & Limit Stencils \\
\hline $\mathrm{A}_{1}$ & $\{0.0027,0.0715,0.2487,0.2127,0.1489,0.0605\}$ \\
\hline $\mathrm{A}_{2}$ & $\{0.0021,0.0840,0.4139,0.4139,0.0840,0.0021\}$ \\
\hline $\mathrm{A}_{3}$ & $\{0.0004,0.0268,0.2389,0.4722,0.2365,0.0263,0.0003\}$ \\
\hline $\mathrm{A}_{4}$ & $\{0.0007,0.0096,0.1024,0.3603,0.3811,0.1299,0.0364$, \\
$0.0084,0.0005\}$
\end{tabular}

TABLE 1. ANALYSIS OF FAMILY OF SUBDIVISION SCHEMES

\begin{tabular}{|c|c|c|c|c|c|c|c|}
\hline $\mathrm{n}$ & $\mathrm{A}_{\mathrm{n}}$ & $\mathrm{GD}$ & $\mathrm{RD}$ & $\mathrm{OC}$ & $\mathrm{OC}_{1 / 2}$ & $\mathrm{GD}_{1 / 2}$ & $\mathrm{HR}_{1 / 2}$ \\
\hline 1 & $\mathrm{~A}_{1}$ & 4 & 1 & $\mathrm{C}^{4}$ & $\mathrm{C}^{5}$ & 5 & 5.1256 \\
\hline 2 & $\mathrm{~A}_{2}$ & 4 & 1 & $\mathrm{C}^{4}$ & $\mathrm{C}^{6}$ & 6 & 6.1256 \\
\hline 3 & $\mathrm{~A}_{3}$ & 4 & 1 & $\mathrm{C}^{4}$ & $\mathrm{C}^{7}$ & 7 & 7.1256 \\
\hline 4 & $\mathrm{~A}_{4}$ & 4 & 1 & $\mathrm{C}^{4}$ & $\mathrm{C}^{8}$ & 8 & 8.1256 \\
\hline 5 & $\mathrm{~A}_{5}$ & 4 & 1 & $\mathrm{C}^{4}$ & $\mathrm{C}^{9}$ & 9 & 9.1256 \\
\hline
\end{tabular}

Mehran University Research Journal of Engineering \& Technology, Volume 38, No. 2, April, 2019 [p-ISSN: 0254-7821, e-ISSN: 2413-7219] 
and

$\beta_{2}=\frac{1}{21 p^{2}-90 p+70}\left(22 p^{2}-44 p-13-\sqrt{p^{4}+92 p^{3}-66 p^{2}+1004 p+169}\right)$

If

$d_{i}^{k}>0, \frac{1}{\lambda} \leq R^{k} \leq \lambda, \forall k \geq 0, k \in Z, i \in Z$

then 5-point binary approximating subdivision scheme

Equation (8) is convexity preserving.

Proof: Using second order divided difference formula

$d_{i}^{k+1}=2^{2 k+1}\left(f_{i-1}^{k+1}-2 f_{i}^{k+1}+f_{i+1}^{k+1}\right)$

By using Equation (11), the scheme Equation (8) become

$d_{2 i}^{k+1}=\frac{d_{i}^{k}}{48}\left[p^{2} d_{i-2}^{k}+\left(21 p^{2}+48 p+1\right) d_{i-1}^{k+1}+\left(-45 p^{2}+70\right) d_{i}^{k}+\left(23 p^{2}-48 p+25\right) d_{i+1}^{k+1}\right]$

$d_{2 i+1}^{k+1}=\frac{1}{48}\left[\left(23 p^{2}+2 p\right) d_{i-1}^{k}+\left(-45 p^{2}+90 p+25\right) d_{i}^{k}+\left(21 p^{2}-90 p+70\right) d_{i+1}^{k}+\left(p^{2}-2 p+1\right) d_{i+2}^{k}\right]$

We use mathematical induction to prove $d_{i}^{k}>0$ and $\frac{1}{\lambda} \leq R^{k} \leq \lambda$. When $\mathrm{k}=0$, it is obvious from statement $d_{i}^{0}>0$ and $\frac{1}{\lambda} \leq R^{0} \leq \lambda$.

$d_{i}^{0}>0$ and $\frac{1}{\lambda} \leq R^{0} \leq \lambda$.
Suppose $d_{i}^{k}>0$ and $\frac{1}{\lambda} \leq R^{k} \leq \lambda$ is true fork. Now we prove for $\mathrm{k}+1$. By re-writing Equation (10) as:

$d_{2 i}^{k+1}=\frac{d_{i}^{k}}{48 \lambda}\left[\lambda^{2}\left(21 p^{2}+48 p+1\right)+\lambda\left(-44 p^{2}+70\right)+\left(23 p^{2}-48 p+25\right)\right]$

$d_{2 i}^{k+1}>0$, for $\beta_{1}<\lambda<\beta_{2}$

Where

$\beta_{1}=\frac{1}{21 p^{2}-90 p+70}\left(22 p^{2}-44 p-13+\sqrt{p^{4}+92 p^{3}-66 p^{2}+1004 p+169}\right)$

and

$\beta_{2}=\frac{1}{21 p^{2}-90 p+70}\left(22 p^{2}-44 p-13-\sqrt{p^{2}+92 p^{2}-66 p^{2}+1004 p+169}\right)$

Now consider Equation (13) to show $d_{2 i+1}^{k+1}>0$

$d_{2 i+1}^{k+1}=\frac{d_{i}^{k}}{48}\left[\left(23 p^{2}+2 p\right) \frac{d_{i-1}^{k}}{d_{i}^{k}}+\left(-45 p^{2}+90 p+25\right) \frac{d_{i}^{k}}{d_{i}^{k}}+\left(21 p^{2}-90 p+70\right) \frac{d_{i+1}^{k}}{d_{i}^{k}}+\left(p^{2}-2 p+1\right) \frac{d_{i+2}^{k}}{d_{i}^{k}}\right]$

This implies

$d_{2 i+1}^{k+1}-\frac{d_{i}^{k}}{48 \lambda}\left[\lambda^{2}\left(21 p^{2}-90 p+70\right)+\left(-44 p^{2}+88 p+26\right) \lambda+\left(23 p^{2}+p\right)\right]$

$d_{2 i+1}^{k+1}>0$, for $\beta_{1}<\lambda<\beta_{2}$ with $\mathrm{p} \in(0,1)$

Thus

$d_{2 i+1}^{k+1}>0, \forall k \geq 0, k \in Z, i \in Z$

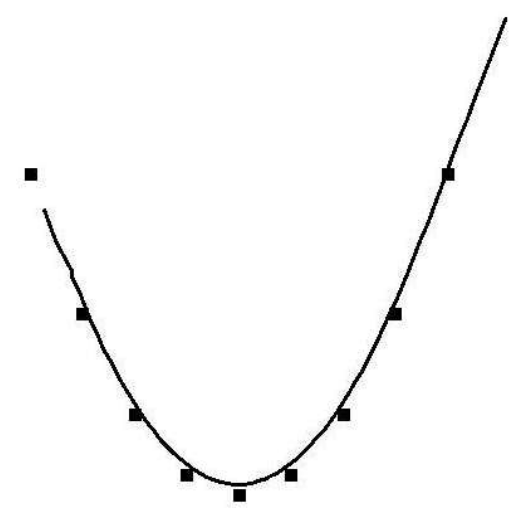

(a) Positively Skewed $A_{2}$ at $\mathrm{p}=1 / 10$

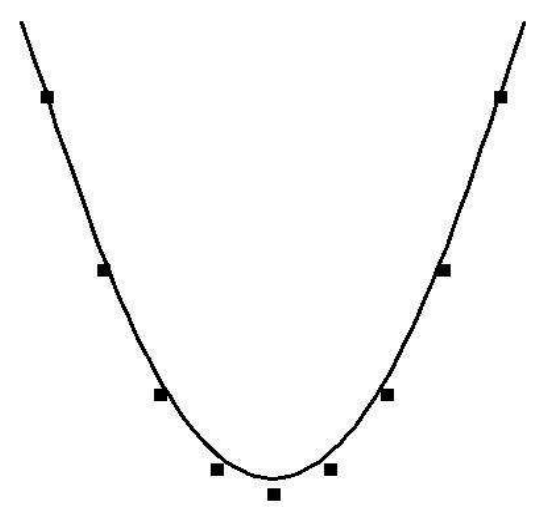

(b) Normal $A_{2}$ at $p=1 / 2$

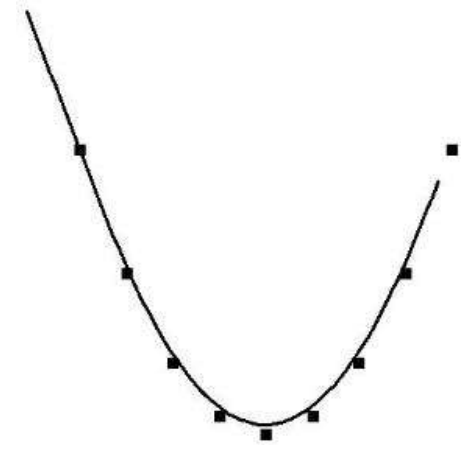

(c) Negatively Skewed $A_{2}$ at $\mathrm{p}=1 / 10$

FIG. 1. PRESENT THE BEHAVIOR OF THE SCHEME $A_{2}$ 
To prove $\frac{1}{\lambda} \leq R^{k+1} \leq \lambda, \forall k \geq 0$ it is sufficient to prove that

$\frac{1}{\lambda}<r_{i}^{k+1}<\lambda, \forall i \in Z, \forall k \geq 0, k \in Z, i \in Z$

Since we know that $r_{2 i}^{k+1}=\frac{d_{2 i+1}^{k+1}}{d_{2 i}^{k+1}}$

$r_{2 i}^{k+1}-\lambda=\frac{d_{2 i+1}^{k+1}}{d_{2 i}^{k+1}}-\lambda=\frac{d_{2 i+1}^{k+1}-\lambda d_{2 i}^{k+1}}{d_{2 i}^{k+1}}=\frac{A}{B}$

where $A=d_{2 i+1}^{k+1}-\lambda d_{2 i}^{k+1}$ and $d_{2 i}^{k+1}$

From Equation (12), we have already proved that $d_{2 i}^{k+1}>0$ So, $B>0$ and the numerator satisfiers

$$
\begin{aligned}
A=\left(23 p^{2}\right. & +2 p) \frac{1}{r_{i-1}^{k}}+\left(-45 p^{2}+90 p+25\right)+\left(21 p^{2}-90 p+70\right) r_{i}^{k}+\left(p^{2}-2 p+1\right) r_{i}^{k} r_{i+1}^{k} \\
& -\lambda p^{2} \frac{1}{r_{i-1}^{k}} \frac{1}{r_{i-2}^{k}}-\lambda\left(21 p^{2}+48 p+1\right) \frac{1}{r_{i-1}^{k}}-\lambda\left(-45 p^{2}+70\right)-\lambda(23 p-48 p+45) r_{i}^{k}
\end{aligned}
$$

This implies

$$
\begin{array}{r}
A=\frac{1}{r_{i-1}^{k}}\left(23 p^{2}+2 p-21 p^{2} \lambda-48 p \lambda-\lambda\right)+r_{i}^{k}\left(21 p^{2}-90 p+70-23 p^{2} \lambda+48 p \lambda-25 \lambda\right) \\
+\frac{1}{r_{i-1}^{k}} \frac{1}{r_{i-2}^{k}}\left(-p^{2} \lambda\right)+r_{i}^{k} r_{i+1}^{k}\left(p^{2}-2 p+1\right)+\left(-45 p^{2}+90 p+25+45 p^{2} \lambda-70 \lambda\right)
\end{array}
$$

This further implies

$$
\begin{gathered}
A=\frac{1}{r_{i-1}^{k}}\left(23 p^{2}+2 p-21 p^{2} \lambda-48 p \lambda-\lambda-p^{2}\right)+r_{i}^{k}\left\{21 p^{2}-90 p+70-23 p^{2} \lambda+48 p \lambda\right. \\
\left.-25 \lambda+\frac{1}{\lambda}\left(p^{2}-2 p+1\right)\right\}+\left(-45 p^{2}+90 p+25+45 p^{2} \lambda-70 \lambda\right)
\end{gathered}
$$

Again implies

$$
\begin{aligned}
& \mathrm{A}=\lambda\left(23 p^{2}+2 p-21 p^{2} \lambda-48 p \lambda-\lambda-p^{2}\right)+\lambda\left\{21 p^{2}-90 p+70\right. \\
& \left.-23 p^{2} \lambda+48 p \lambda-25 l+1 / \lambda\left(p^{2}-2 \mathrm{p}+1\right)\right\}+\left(45 p^{2}+90 p+25\right. \\
& \left.+45 p^{2} \lambda-70 \lambda\right)
\end{aligned}
$$

Furthermore

$A=\lambda^{2}\left(-44 p^{2}-26\right)+\lambda\left(88 p^{2}-88 p\right)+\left(-44 p^{2}-88 p+26\right)$
This implies $A<0$ for $b_{1}<l<b_{2}$. Therefore, $r_{2 i}^{k+1}<\lambda, \forall \mathrm{i} \in \mathrm{Z}$.

Similarly

$$
\frac{1}{r_{2 i}^{k+1}}-\lambda=\frac{d_{2 i}^{k+1}}{d_{2 i+1}^{k+1}}-\lambda=\frac{d_{2 i}^{k+1}-\lambda d_{2 i+1}^{k+1}}{d_{2 i+1}^{k+1}}=\frac{C}{D}
$$

where $C=d_{2 i}^{k+1}-\lambda d_{2 i+1}^{k+1}$ and $D=d_{2 i+1}^{k+1}$

From Equation (13), we have already proved that $d_{2 i+1}^{k+1}>0$ so, $D>0$ and the numerator satisfies

$$
\begin{aligned}
& C=p^{2}+\left(21 p^{2}+48 p+1\right) r_{i-2}^{k}+\left(-45 p^{2}+70\right) \\
& r_{i-2}^{k} r_{i-1}^{k}+\left(23 p^{2}-4 p+25\right) r_{i-2}^{k} r_{i-1}^{k} r_{i}^{k}-\lambda\left(23 p^{2}+p\right) \\
& r_{i-2}^{k}-\lambda\left(45 p^{2}+90 p+25\right) r_{i-2}^{k} r_{i-1}^{k}-\lambda\left(21 p^{2}-90 p+70\right) \\
& r_{i-2}^{k} r_{i-1}^{k} r_{i}^{k}-\lambda\left(p^{2}-2 p+1\right) r_{i-2}^{k} r_{i-1}^{k} r_{i}^{k} r_{i+1}^{k}
\end{aligned}
$$

This implies

$$
\begin{aligned}
& C=p^{2}+\left(21 p^{2}+48 p+1-23 p^{2} \lambda-2 p \lambda\right) \\
& r_{i-2}^{k}+\left(-45 p^{2}+70+45 p^{2} \lambda-90 p \lambda-25 \lambda\right) \\
& r_{i-2}^{k} r_{i-1}^{k}+\left(23 p^{2}-4 p+25-21 p^{2} \lambda+90 p \lambda-70 \lambda\right) \\
& r_{i-2}^{k} r_{i-1}^{k} r_{i}^{k}-\lambda\left(p^{2}-2 p+1\right) r_{i-2}^{k} r_{i-1}^{k} r_{i+1}^{k}
\end{aligned}
$$

This again implies

$$
\begin{aligned}
C= & p^{2}+\left(21 p^{2}+48 p+1-23 p^{2} \lambda-2 p \lambda\right) \\
r_{i-2}^{k}+ & \left(-25 p^{2}+70+45 p^{2} \lambda-90 p \lambda-25 \lambda+23 p^{2} \lambda-4 p \lambda+\right. \\
& \left.25 \lambda-21 p^{2} \lambda^{2}+90 p \lambda^{2}-70 \lambda^{2}-p^{2} \lambda+2 p \lambda-\lambda\right) r_{i-2}^{k} r_{i-1}^{k}
\end{aligned}
$$

Further implies

$C=p^{2}+\lambda\left(21 p^{2} \lambda+48 p \lambda+1-23 p^{2} \lambda^{2}-2 p \lambda^{2}-45 p^{2}+70+\right.$ $45 p^{2} \lambda-90 p \lambda-251+23 p^{2} \lambda-4 p \lambda+251-21 p^{2} \lambda^{2}+90 p \lambda^{2}-$ $\left.70 \lambda^{2}-p^{2} \lambda+2 p \lambda-\lambda\right)$

Furthermore

$C=\lambda^{2}\left(-44 p^{2}+88 p-70\right)+\lambda\left(88 p^{2}-88 p\right)+\left(-44 p^{2}+70\right)$ 
This implies $C<0$ for $\beta_{1}<\lambda<\beta_{2}$.

Thus $\frac{1}{r_{2 i}^{k+1}}-\lambda=0$. Combining Equations (16) and Equation (17), it can be written as:

$\frac{1}{\lambda} \leq r_{2 i}^{k+1} \leq \lambda, \forall i \in Z$

Similarly, we can show that

$\frac{1}{\lambda} \leq r_{2 i+1}^{k+1} \leq \lambda, \forall i \in Z$

Hence $d_{i}^{k}>0$ and $\frac{1}{\lambda} \leq R^{k} \leq \lambda$ is satisfied for $\mathrm{k}+1$. Hence proposed scheme $A_{2}$ preserves convexity. This completes the proof.

\section{COMPARISON AND APPLICATIONS}

In this section, we present the comparison and applications of the proposed family of schemes.

\subsection{Comparison of Continuity Analysis}

Here we present the comparison of continuity analysis of the proposed family of schemes with existing parametric subdivision schemes. It is clear from Table 3 that our proposed family gives higher continuity comparative to the existing parametric subdivision schemes.

Table 3 shows the comparison of continuity analysis. Here, $E, O C, A_{n}$ and $O C_{1 / 2}$ denote the existing schemes, order of continuity of existing schemes, proposed family of schemes and continuity of proposed schemes at $p=1$ / 2 respectively.

\subsection{Applications}

Here we discuss the visual performance of the proposed family of subdivision schemes. The control polygons are drawn by doted lines and the smooth curves obtained by our proposed schemes by full lines. Fig. 2(a-c) represents the applications of proposed scheme $A_{2}$ at $p=1 / 64,1 / 2$ and 9/10. Fig. 3(a-f) is the applications of scheme $A_{2}$ at different values of $p$.

TABLE 3. COMPARISON TABLE

\begin{tabular}{|c|c|c|c|}
\hline $\mathrm{E}$ & $\mathrm{OC}$ & $A_{n}$ & $\mathrm{OC}_{1 / 2}$ \\
\hline 5-Point [20] & $\mathrm{C}^{2}$ if $\frac{1}{324}<\mathrm{w}<\frac{1}{162}$ & \multirow{6}{*}{$\mathrm{A}_{2}$} & \multirow{6}{*}{$\mathrm{C}^{6}$} \\
\hline 5-Point [8] & $\mathrm{C}^{4}$ & & \\
\hline 5-Point [13] & $\mathrm{C}^{5}$ & & \\
\hline 5-Point [9] & $\mathrm{C}^{2}$ if $\frac{-3}{64}<\mathrm{w}<\frac{1}{162}$ & & \\
\hline 5 -Point [21] & $\mathrm{C}^{4}$ & & \\
\hline 6-Point [22] & $\mathrm{C}^{7}$ & & \\
\hline 6-Point [10] & $\mathrm{C}^{2}$ if $\mathrm{w} \in[0,0.0425]$ & \multirow{6}{*}{$\mathrm{A}_{4}$} & \multirow{6}{*}{$\mathrm{C}^{8}$} \\
\hline 6-Point [10] & $\mathrm{C}^{3}$ if $\mathrm{w} \in[0.0139,0.0143]$ & & \\
\hline 6-Point [11] & $\mathrm{C}^{6}$ & & \\
\hline 6-Point [12] & $\mathrm{C}^{2}$ if $\frac{7}{972}<\mathrm{w}<\frac{11}{1215}$ & & \\
\hline 6-Point [23] & $\mathrm{C}^{3}$ & & \\
\hline 6-Point [24] & $\mathrm{C}^{4}$ & & \\
\hline
\end{tabular}

Mehran University Research Journal of Engineering \& Technology, Volume 38, No. 2, April, 2019 [p-ISSN: 0254-7821, e-ISSN: 2413-7219] 


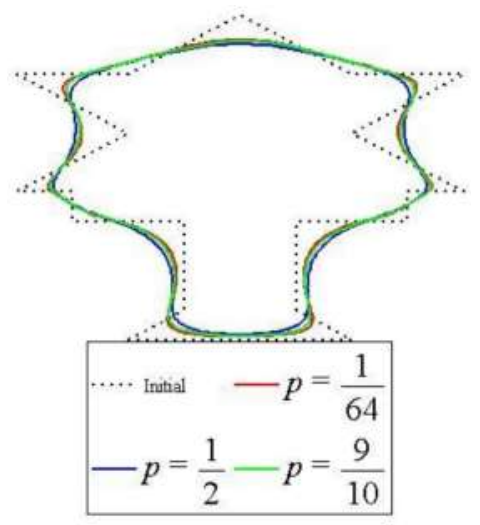

(a)

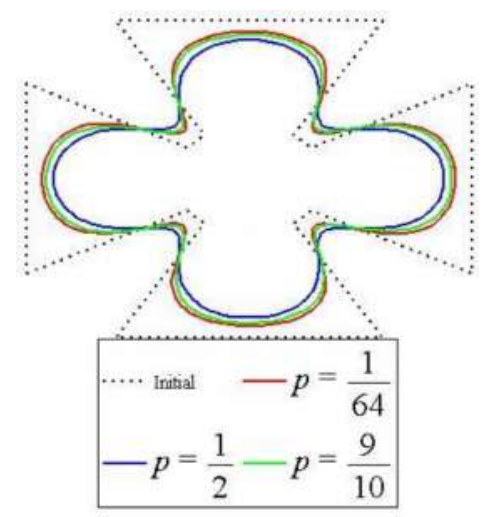

(b)

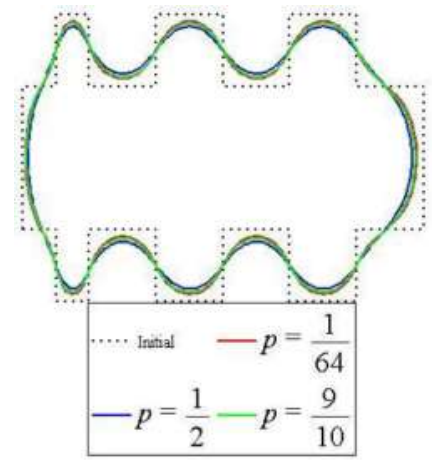

(c)

FIG. 2. PRESENT COMPARISON OF THE LIMIT CURVES FOR CLOSE POLYGONS PRODUCED BY THE SCHEME CORRESPONDING TO $\mathrm{A}_{2}$

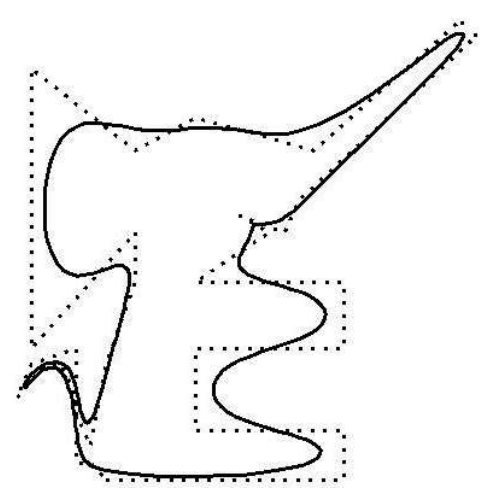

(a) $p=1 / 10$

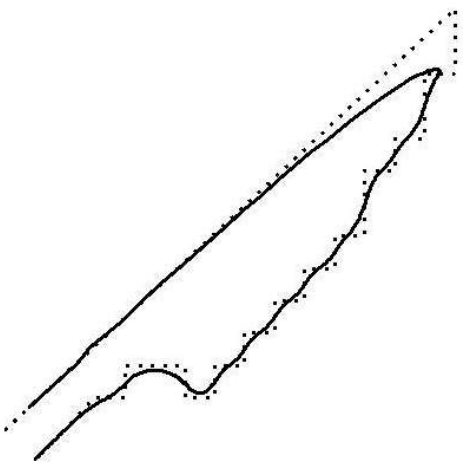

(d) $p=2 / 9$

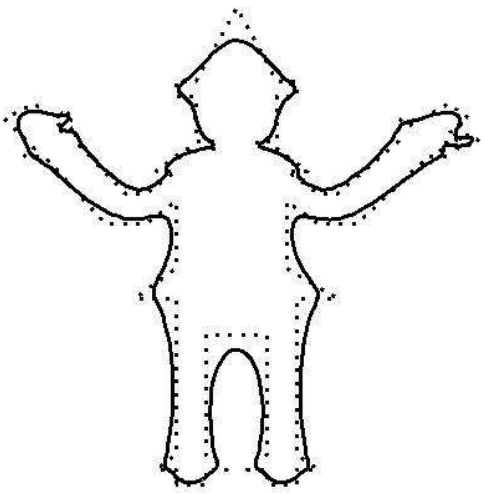

(b) $p=9 / 10$

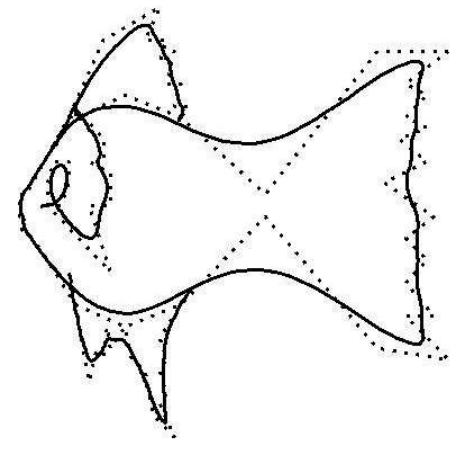

(c) $p=1 / 2$

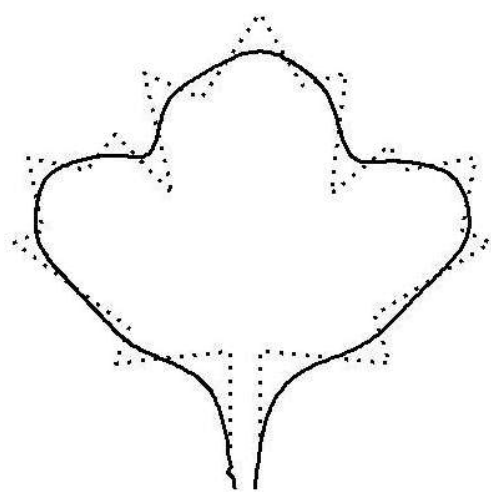

(e) $p=1 / 2$

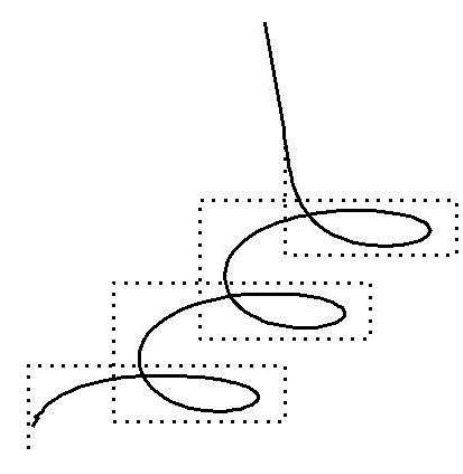

(f) $p=1 / 10$

FIG. 3. PRESENT LIMIT CURVES FOR OPEN AND CLOSE POLYGONS PRODUCED BY THE SCHEME CORRESPONDING TO $A_{2}$

\section{CONCLUSION}

In this paper, Binomial probability distribution is used for the construction of new family of binary approximating subdivision schemes. A complete analysis of some family members of the proposed family is presented. We also analyzed that our proposed family give high continuity at $p=1 / 2$ comparative to the existing subdivision schemes. At all other values of probability parameter $p$ the results of subdivision schemes remain same. 
The parametrization of the subdivision schemes changes at different values of $p$. Subdivision schemes give skewed behavior on the convex data due to the probability parameter. Visual performances of the proposed family of subdivision schemes are also discussed.

\section{ACKNOWLEDGEMENT}

This work is supported by NRPU (National Research Program for Universities) P. No. 3183, Pakistan.

\section{REFERENCES}

[1] Rham,de. G., "Un Peu De Mathématiques à Propos D'uneCourbe Plane", Elemente Der Mathematik, Volume 2, pp. 73-76, 1947.

[2] Chaikin, G.M., "An Algorithm for High-Speed Curve Generation", Computer Graphics and Image Processing, Volume 3, No.4, pp. 346-349, 1974.

[3] Dyn,N., Gregory, J.A., and Levin, D., "A 4-Points Interpolatory Subdivision Scheme for Curve Design", Computer Aided Geometric Design, Volume 4, pp. 257-268, 1987.

[4] Mustafa, G., and Liu, X.F., "A New Solid Subdivision Scheme", Journal of University of Science and Technology of China, Volume 35, No.3, pp. 297-311, 2005.

[5] Mustafa, G., Khan, F., and Ghaffar, A., "The $m$-Point Approximating Subdivision Scheme", Lobachevskii Journal of Mathematics, Volume 30, No. 2, pp. 138-145, 2009.

[6] Ghaffar, A. and Mustafa, G., "A Family of Even-Point Ternary Approximating Schemes", ISRN Applied Mathematics, Article ID 197383, Volume 14, 2012.

[7] Mustafa, G., Ghaffar, A., and Bari, M., "(2n-1)-Point Binary Approximating Scheme”, Proceeding of IEEE $8^{\text {th }}$ International Conference on Digital Information Management, pp. 363-368, 2013.

[8] Ashraf, P., Mustafa, G., and Deng, J., “A 6-Point Variant on the Lane-Riesenfeld Algorithm", Journal of Applied Mathematics, Volume 7, 2014.

[9] Cao, H., and Tan, J., "A Binary 5-Point Relaxation Subdivision Scheme", Journal of Information and Computational Science, Volume 10, No. 18, pp. 5903-5910, 2013.

[10] Mustafa, G., Khan, F., and Hashmi, M.S., “A Generalized Proof of the Smoothness of 6-Point Interpolatory Scheme", Journal of Information and Computing Science, Volume 5, No. 4, pp. 299-304, 2010.
Bari, M., Bashir, R., and Mustafa, G., "3n-Point Quaternary Shape Preserving Subdivision Schemes", Mehran University Research Journal of Engineering \& Technology, Volume 36, No. 3, pp. 489-500, Jamshoro, Pakistan, July, 2017.

[12] Siddiqi, S.S., and Noreen, T., "Convexity Preservation of 6-Point $\mathrm{C}^{2}$ Interpolating Subdivision Scheme", Applied Mathematics and Computation, pp. 936-944, 2015.

[13] Tan, J., Yao, Y., Cao, H., and Zhang, L., "Convexity Preservation of 5-Point Binary Subdivision Scheme with a Parameter", Applied Mathematics and Computation, Volume 245, pp. 279-288, 2014.

[14] Zheng, H.C., Huang, S.P., Guo, F., and Peng, G.H., "Designing Multi-Parameter Curve Subdivision Schemes with High Continuity", Applied Mathematics and Computation, Volume 243,pp. 197-208, 2014.

[15] Bernoulli, J., "ArsConjectandi", Opus Posthumum. Accedit Tractatus de Seriebusinfinitis, et Epistola Gallice Scripta de Ludo Pilae Recticularis, Impensis Thurnisiorum, Fratrum, Basel, 1713.

[16] Siddiqi, S.S., and Younis,M., "Construction of $m$-Point Binary Approximating Subdivision Schemes", Applied Mathematics Letters, Volume 26, pp.337-343, 2013.

[17] Walck, C., "Handbook on Statistical Distributions for Experimentalists", University of Stockholm, 2007.

[18] Hormann, K., "Subdivision Schemes for Geometric Modeling a Hands-on Tutorial", Dolomites Research Notes on Approximation, [ISSN: 2035-6803], University of Verona, 2012.

[19] Rioul, O., "Simple Regularity Criteria for Subdivision Schemes", SIAM Journal on Mathematical Analysis, Volume 23, No. 6, pp. 1544-1576, 1992.

[20] Mustafa, G., Irum, J., and Bari, M., "A New 5-Point Ternary Interpolating Subdivision Scheme and its Differentiability", ISRN Computational Mathematics, 2012.

[21] Lu, Q., Tan, J., and Shi, J., "A Unified Ternary Curve Subdivision Scheme", Journal of Mathematical Research with Applications, Volume 37, No. 1, pp. 47-58, 2017.

[22] Mustafa, G., and Ghaffar, A., "A Family of Even-Point Ternary Approximating Schemes", ISRN Computational Mathematics, 2012.

[23] Rehan, K., and Siddiqi, S.S., "A Combined Binary 6Point Subdivision Scheme", Applied Mathematics and Computation, Volume 270, pp. 130-135, 2015.

[24] Jin, H.Z., "Continuity Analysis and Construction of Uniform Stationary Univariate Subdivision Schemes", Journal of Software, Volume 17, No. 3, pp. 559-567, 2006. 